\title{
Reference values for intracranial pressure and lumbar cerebrospinal fluid pressure: a systematic review
}

\author{
Nicolas Hernandez Norager ${ }^{1 *} \mathbb{D}$, Markus Harboe Olsen², Sarah Hornshoej Pedersen ${ }^{1}$, Casper Schwartz Riedel ${ }^{1,3}$, \\ Marek Czosnyka ${ }^{4}$ and Marianne Juhler ${ }^{1,5}$
}

\begin{abstract}
Background: Although widely used in the evaluation of the diseased, normal intracranial pressure and lumbar cerebrospinal fluid pressure remain sparsely documented. Intracranial pressure is different from lumbar cerebrospinal fluid pressure. In addition, intracranial pressure differs considerably according to the body position of the patient. Despite this, the current reference values do not distinguish between intracranial and lumbar cerebrospinal fluid pressures, and body position-dependent reference values do not exist. In this study, we aim to establish these reference values.

Method: A systematic search was conducted in MEDLINE, EMBASE, CENTRAL, and Web of Sciences. Methodological quality was assessed using an amended version of the Joanna Briggs Quality Appraisal Checklist. Intracranial pressure and lumbar cerebrospinal fluid pressure were independently evaluated and subdivided into body positions. Quantitative data were presented with mean \pm SD, and $90 \%$ reference intervals.

Results: Thirty-six studies were included. Nine studies reported values for intracranial pressure, while 27 reported values for the lumbar cerebrospinal fluid pressure. Reference values for intracranial pressure were -5.9 to $8.3 \mathrm{mmHg}$ in the upright position and 0.9 to $16.3 \mathrm{mmHg}$ in the supine position. Reference values for lumbar cerebrospinal fluid pressure were 7.2 to $16.8 \mathrm{mmHg}$ and 5.7 to $15.5 \mathrm{mmHg}$ in the lateral recumbent position and supine position, respectively.

Conclusions: This systematic review is the first to provide position-dependent reference values for intracranial pressure and lumbar cerebrospinal fluid pressure. Clinically applicable reference values for normal lumbar cerebrospinal fluid pressure were established, and are in accordance with previously used reference values. For intracranial pressure, this study strongly emphasizes the scarcity of normal pressure measures, and highlights the need for further research on the matter.
\end{abstract}

Keywords: Cerebrospinal fluid pressure, CSF, ICP, Intracranial pressure, Reference intervals, Reference values

\section{Background}

Measurements and analysis of intracranial pressure (ICP) are used to provide information for treating and managing patients with numerous neurological diseases, e.g.,

\footnotetext{
*Correspondence: nicolas.norager@gmail.com

${ }^{1}$ Department of Neurosurgery, Rigshospitalet, Copenhagen, Denmark

Full list of author information is available at the end of the article
}

traumatic brain injury, acute intracranial hemorrhages, idiopathic intracranial hypertension, and hydrocephalus [1-4]. However, values for normal ICP measured intracranially remain sparsely documented, and the currently used reference interval (7 to $15 \mathrm{mmHg}$ [5]) is based on studies in which lumbar cerebrospinal fluid opening pressure $\left(\mathrm{LCSF}_{\mathrm{op}}\right)$ is used as a surrogate parameter for

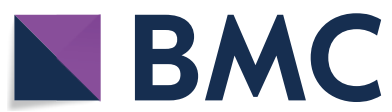

(c) The Author(s) 2021. This article is licensed under a Creative Commons Attribution 4.0 International License, which permits use, sharing, adaptation, distribution and reproduction in any medium or format, as long as you give appropriate credit to the original author(s) and the source, provide a link to the Creative Commons licence, and indicate if changes were made. The images or other third party material in this article are included in the article's Creative Commons licence, unless indicated otherwise in a credit line to the material. If material is not included in the article's Creative Commons licence and your intended use is not permitted by statutory regulation or exceeds the permitted use, you will need to obtain permission directly from the copyright holder. To view a copy of this licence, visit http://creativeco mmons.org/licenses/by/4.0/. The Creative Commons Public Domain Dedication waiver (http://creativecommons.org/publicdomain/ zero/1.0/) applies to the data made available in this article, unless otherwise stated in a credit line to the data. 
ICP or is extrapolated from patients with a suspected ICP disorder [5-10].

In the $1920 \mathrm{~s}$ and $30 \mathrm{~s}$, it was assumed that ICP measured intracranially was equal to $\operatorname{LCSF}_{\text {op }}$ measured at a lumbar spine level $[11,12]$. This has, in recent years, been subject to much debate [13-17], and where some studies have documented similar values for intracranial measurements and lumbar measurements $[13,15]$, others found considerable differences between the two measurement-sites [14, 16, 17]. The reason for a difference might be due to ICP being measured in the brain parenchyma, thus not being measured in the same intracranial space as LCSFop. Thus, ICP measured ventricular might similar to LCSFop, although this has yet to be thoroughly investigated. The comparison of these studies is further hampered by differences in study design, particularly dissimilarities in body position during the measurement and the range of included study participants [13-16, 18, 19]. It is well known that ICP changes with body position $[8,18-21]$, e.g., ICP is lower in the vertical position compared to the horizontal position $[8,19,21]$. Also, ICP increases significantly with the body placed in lateral recumbent position compared to supine position [18, 22-24]. Based on this, different reference values for ICP should be established for different body positions. This systematic review aims to (1) determine reference values for normal ICP and $\mathrm{LCSF}_{\mathrm{op}}$, and (2) examine how ICP depends on measurement-site and body position.

\section{Methods}

Prior to initiating this systematic review, the protocol was registered at PROSPERO (October 30, 2019, identification code: CRD42019143018). The review has been conducted in accordance with the PRISMA guidelines, and a completed PRISMA checklist is available in Additional file 1: Appendix 1.

\section{Eligibility criteria}

All included studies had to provide (1) measurements conducted in humans, (2) original values for either ICP or $\mathrm{LCSF}_{\mathrm{op}}$, and (3) ICP measured either intracranially (parenchymal, intraventricular, epidural, subdural, or subarachnoid) or LCSF $_{\text {op }}$ measured during a lumbar puncture. All age-groups were included. Patients with intracranial pathology that potentially could alter ICP dynamics (e.g., hydrocephalus, idiopathic intracranial hypertension, intracranial hemorrhage, arteriovenous malformation, intracranial tumor, intracranial abscesses or other significant space-occupying processes within the brain) were excluded.
Since measurement of ICP requires invasive measurement methods, the included studies of ICP are based on "pseudo-healthy" patients, which we define as patients requiring neurosurgery for reasons unrelated to ICP, who were considered healthy in terms of ICP and CSF-dynamics.

\section{Data search}

On July 16st 2019, we conducted a search in MEDLINE Ovid (1946 to July 2019), Embase Ovid (1974 to July 2019), Cochrane Central Register of Controlled Trials (CENTRAL) in the Cochrane Library and Science Citation Index Expandex (1900 to July 2019), and Conference Proceeding Citation Index-Science (Web of Science) (1990 to July 2019). There was no restriction on the publication period. We included all peer-reviewed published studies without consideration for publication status or study design. The search was conducted in English, and studies with a non-English title or abstract were thus excluded. Non-English manuscripts, with an English title and abstract, were translated by a native speaker. The search strategy is attached in Additional file 2: Appendix 2. If published data in the included studies were insufficient, the authors were contacted to retrieve raw data. The systematic search was supplemented by a manual reference-search of included studies. Finally, an expert in the field (MJ) was asked to identify any obviously missing studies.

\section{Study selection and data extraction}

Four investigators (CSR, MHO, NHN, SHP) reviewed the studies for eligibility. Two different investigators assessed each study for the title, abstract and full-text screening. Discrepancies were initially resolved between the four investigators, and if this was not possible, an expert (MJ) had the deciding vote. The screening was conducted via Covidence (Covidence systematic review software, Veritas Health Innovation, Melbourne, Australia). Study design, demographics, and information regarding ICPmonitoring were extracted from each study. Study data were extracted from Covidence by NHN and verified by MHO. Disagreements were resolved by discussion.

\section{Quality assessment}

The methodological quality of the studies was independently assessed by two investigators (MHO, NHN). No intervention-based studies were included, and thus, traditional assessment methods for systematic reviews could not be used. Instead, a quality assessment method was created inspired by the Joanna Briggs Institute Critical Appraisal Tool [25]. Two parts of the tool were, however, 
irrelevant in the assessment of study type and therefore replaced; (1) "Was the exposure measured in a valid and reliable way?" was replaced with "Was the measurement method described in details?" and (2) "Were the outcomes measured in a valid and reliable way?" was replaced with "Was the included population without current or previous intracranial pathology that could alter ICP dynamics?”.

\section{Data synthesis}

All statistical analyses were performed in RStudio ( $R$ 3.6.2, R Development Core Team (2019), Vienna, Austria). Since all included studies were observational, and the primary purpose of this review was to determine a reference interval for ICP, a conventional meta-analysis was not applicable. Instead, included studies were pooled into two groups, (1) studies in which ICP was measured, and (2) studies in which LCSF $_{\text {op }}$ was measured. Raw data were assessed for normality and presented as mean \pm standard deviation (SD), 95\% confidence intervals $(\mathrm{CI})$, and coherent reference intervals (defined as 5 th to 95th percentile) [5]. If statistical values other than SD were presented (e.g., 95\% confidence intervals or reference intervals), SD was manually calculated based on the given statistical data according to the Cochrane Handbook of Systematic Reviews [26]. For studies where only the median and interquartile range (IQR) were presented, the median was directly transformed to a mean, while $\mathrm{SD}$ was estimated from $\mathrm{IQR}$ by $\mathrm{SD}=\mathrm{IQR} / 1.35$, as recommended in the Cochrane Handbook of Systematic Reviews [26]. Finally, if pressure values were reported in other units (e.g., $\mathrm{mmH}_{2} \mathrm{O}$ or $\mathrm{cmH}_{2} \mathrm{O}$ ) it was converted to mmHg.

Normal ICP and LCSF $_{\text {op }}$ were subdivided into different body positions stratified by study and presented in a forest plot, including mean values and $95 \% \mathrm{CI}$. The corresponding weighted reference intervals for the different body positions were subsequently presented in a table for ICP and $\mathrm{LCSF}_{\mathrm{op}}$. Pressure measurements are presented in both $\mathrm{mmHg}$ and $\mathrm{cmH}_{2} \mathrm{O}$. The different body positions were compared using a two-tailed Students t-test, and P-values were presented. Due to multiplicity, we chose to Bonferroni-correct in comparison of body positions and thereby lowering the risk of type 1 errors. P-values $<0.05$ were considered significant. The included studies provided insufficient data to perform a meaningful multivariate analysis adjusting for age, gender, monitoring equipment, neck position, and zero point for ICP measurement-site.

\section{Results}

The search strategy identified a total of 2516 studies. Of these, six were found from other sources than our search string. All these six studies were found by manually searching reference list of included studies. After the removal of duplicates, 1791 studies remained. The abstract and title screening left 127 studies, and based on full-text screening, 44 studies were included (Fig. 1). Study characteristics are presented in Table 1.

The majority of studies reported a mean value with coherent SD or a mean value with either $95 \%$ CI or reference intervals, from which SD could be calculated $(\mathrm{n}=32)$. In a few studies, only median and IQR were reported $(n=4)$. Studies that only reported a range or a mean and no further statistical data could be provided from the authors were omitted from analysis $(n=8)$. Thus, 36 studies remained in the data analysis.

\section{Reference intervals for ICP and LCSF ${ }_{\text {op }}$}

Reference intervals differed significantly between body positions (e.g., lateral recumbent position $\mathrm{LCSF}_{\mathrm{op}}$ versus supine $\operatorname{LCSF}_{\text {op }}(\mathrm{P}=0.04)$, and upright ICP versus supine ICP $(\mathrm{P}<0.01))$. Details are shown in Table 2 for both ICP and LCSF $_{\text {op }}$. Nine studies provided values for ICP measured intracranially. Reference intervals were subdivided into four groups based on body position: (1) supine position with a mean ICP of $8.6 \mathrm{mmHg}$ (SD 4.7, reference interval 0.9 to $16.3 \mathrm{mmHg}$ ), (2) upright position with a mean ICP of $1.0 \mathrm{mmHg}$ (SD 4.3, reference interval -5.9 to $8.3 \mathrm{mmHg}$ ), (3) continuous daytime measurement with a mean ICP of $-0.1 \mathrm{mmHg}$ (SD 7.4, reference interval -12.0 to $12.2 \mathrm{mmHg}$ ), and (4) continuous nighttime measurement with a mean ICP of $6.3 \mathrm{mmHg}$ (SD 13.3, reference interval -15.8 to 28.2) (Fig. 2, Table 2).

Twenty-seven studies provided values for $\mathrm{LCSF}_{\mathrm{op}}$ measured by lumbar puncture. LCSF $_{\text {op }}$ was subdivided into two groups: (1) the supine position with a mean $\mathrm{LCSF}_{\text {op }}$ of $10.7 \mathrm{mmHg}$ (SD 3.0, reference interval 5.7 to $15.5 \mathrm{mmHg}$ ), and (2) the lateral recumbent position with a mean $\mathrm{LCSF}_{\text {op }}$ of $11.9 \mathrm{mmHg}$ (SD 2.9, reference interval 7.2 to $16.8 \mathrm{mmHg}$ ) (Fig. 2, Table 2). There was a statistical difference between ICP values in the supine position and $\mathrm{LCSF}_{\mathrm{op}}$ values in the supine position $(\mathrm{P}=0.03)$. There was no statistical difference between reference intervals for $\operatorname{LCSF}_{\text {op }}$ based solely on the healthy population compared to reference intervals for $\mathrm{LCSF}_{\mathrm{op}}$ based on the pseudo-healthy population. The difference between healthy individuals and pseudo-healthy individuals in lateral recumbent position was $1.4 \mathrm{mmHg}(\mathrm{P}=0.34)$ and in supine position $1.0 \mathrm{mmHg}(\mathrm{P}=0.73)$. 


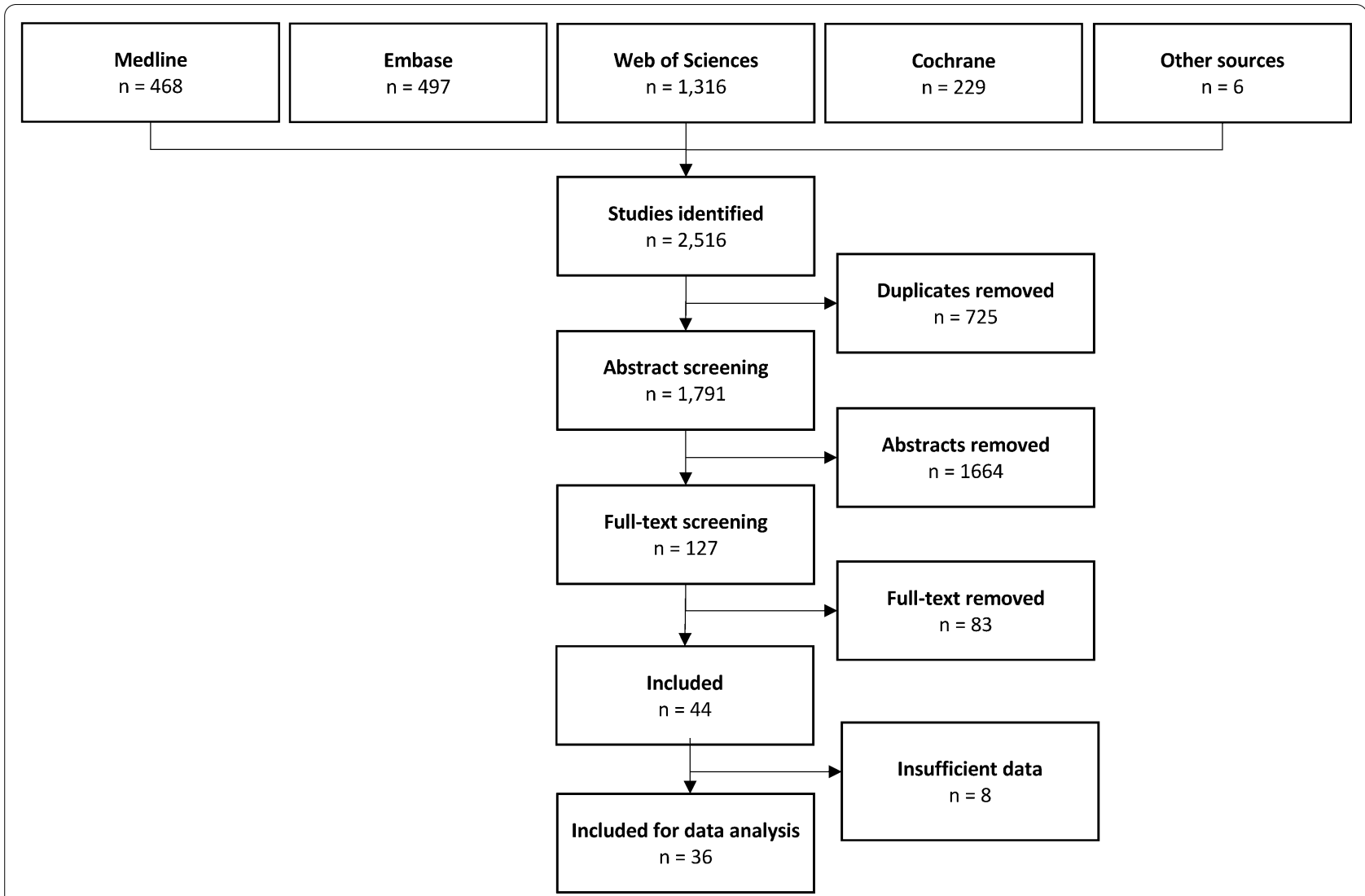

Fig. 1 Flowchart of study selection process. The section "insufficient data" covers studies did not report sufficient statistical data to be included in statistical analysis. Thus, no standard deviations, confidence intervals or reference intervals were reported in these studies. The included articles "Other sources" were found by manual searching the reference list of included studies

\section{Quality of included studies}

The overall methodological quality of the study designs was moderate, see Additional file 3: Appendix 3. Out of seven, the average score of the included studies was 4.5. None of the studies in which ICP was measured were based on completely healthy individuals, and in only two studies, the primary aim was to determine reference intervals for ICP. For the studies in which ICP was measured, the overall methodological study quality was moderate (average study quality $=4 / 7$ ). In the group of $\mathrm{LCSF}_{\text {op }}$, the overall study quality was generally higher (average study quality $=5 / 7$ ) and a few studies included completely healthy individuals $(\mathrm{n}=13)$ [5, 6, 23, 27-35]. In general, studies with $\mathrm{LCSF}_{\text {op }}$ consisted of a significantly higher number of study participants (Table 1 ).

\section{Discussion}

This systematic review aimed to establish reference intervals for normal ICP and $\mathrm{LCSF}_{\mathrm{op}}$. Through an extensive literature search, 44 studies examining either ICP or LCSF in pseudo-healthy or normal individuals were identified, and of these, 36 provided sufficient pressure values and statistical data to calculate reference intervals. Nine studies reported ICP values, while 27 reported LCSF $_{\text {op }}$ values. The overall study quality was moderate. For ICP, the study material was scarce, and the reference intervals were based on small sample sizes. Data on $\mathrm{LCSF}_{\text {op }}$ were, in comparison, comprehensive in both lateral recumbent position and supine position. Finally, the reference intervals for both ICP and $\mathrm{LCSF}_{\text {op }}$ were found to differ significantly between body positions, demonstrating the need for position-dependent reference intervals. After completion of the the present study, $\mathrm{B} \varnothing$ et al. reported a mean $\mathrm{CSF}_{\text {op }}$ of $17.5 \mathrm{cmH}_{2} \mathrm{O}(12.9 \mathrm{mmHg})$ ranging for $4-30 \mathrm{cmH}_{2} \mathrm{O}(2.9-22.1 \mathrm{mmHg})$ in an prospecitvely sampled out-patient population. These results further supports our conclusions [36].

The literature further indicates that age, weight, neck position, zero point for measurement-site, and monitoring equipment are of great importance in measuring ICP and $\operatorname{LCSF}_{\text {op }}[9,23,37-42]$. Especially zero point is of uttermost importance when measuring ICP in upright position. In supine and lateral recumbent position, zeropoint matters to the extent that it has to be in the patient's 
Table 1 Characteristics of included studies

\begin{tabular}{|c|c|c|c|c|c|c|}
\hline & Type of study & Type of population & Measurement place & No. of participant & Mean age or range & $\begin{array}{l}\text { Included } \\
\text { in } \\
\text { analysis }^{\mathrm{a}}\end{array}$ \\
\hline Albeck [33] & Prospective, observational & Healthy & Lumbar & 8 & $22-28$ & Yes \\
\hline Albeck [34] & Prospective, observational & Healthy & Lumbar & 52 & 60 & Yes \\
\hline Andresen [46] & Prospective, observational & Pseudo-healthy & Parenchymal & 4 & 67 & Yes \\
\hline Avery [63] & Prospective, observational & Pseudo-healthy & Lumbar & 197 & $1-18$ & Yes \\
\hline Avery [70] & Case report, literature review & Pseudo-healthy & Lumbar & 1 & 12 & Yes \\
\hline Beck [66] & Prospective, observational & Pseudo-healthy & Lumbar & 17 & - & Yes \\
\hline Blomquist [73] & Prospective, observational & Pseudo-healthy & Lumbar & 18 & $0-15$ & Yes \\
\hline Bono [67] & Prospective, observational & Pseudo-healthy & Lumbar & 111 & 40 & Yes \\
\hline $\mathrm{B} \varnothing[69]$ & Prospective, observational & Pseudo-healthy & Lumbar & 348 & 47 & Yes \\
\hline Chiari [76] & Retrospective, observational & Pseudo-healthy & Parenchymal & 41 & 43 & Yes \\
\hline Chapman [21] & Prospective, observational & Pseudo-healthy & Ventricular & 5 & 28 & Yes \\
\hline Corbett [25] & Prospective, observational & Healthy & Lumbar & 15 & - & Yes \\
\hline Eklund [32] & Prospective, observational & Healthy & Lumbar & 11 & 46 & Yes \\
\hline Ekstedt [9] & Prospective, observational & Pseudo-healthy & Lumbar & 100 & - & Yes \\
\hline Ellis [24] & Prospective, observational & Pseudo-healthy & Lumbar & 33 & 8 & Yes \\
\hline Fleischman [68] & Retrospective, observational & Pseudo-healthy & Lumbar & 12,118 & 54 & Yes \\
\hline Friden [72] & Retrospective, observational & Pseudo-healthy & Lumbar & 150 & - & Yes \\
\hline Gilland [8] & Retrospective, observational & Healthy & Lumbar & 15 & 25 & Yes \\
\hline Gilland [7] & Prospective, observational & Healthy & Lumbar & 31 & 23 & Yes \\
\hline Gonzalez [62] & Prospective, case-control & Pseudo-healthy & Lumbar & 28 & 41 & No \\
\hline Hannerz [35] & Prospective, observational & Healthy & Lumbar & 19 & 52 & Yes \\
\hline Kaiser [60] & Prospective, observational & Pseudo-healthy & Lumbar & 49 & 30 (24-41) weeks & Yes \\
\hline Kawasaki [61] & Retrospective, case-control & Pseudo-healthy & Lumbar & 1 & 51 & Yes \\
\hline Lakke [36] & Prospective, observational & Healthy & Lumbar & 34 & - & No \\
\hline Langvatn [77] & Retrospective, observational & Pseudo-healthy & Parenchymal & 12 & 3 & Yes \\
\hline Lawley [56] & Prospective, observational & Pseudo-healthy & Ventricular & 8 & 35 & Yes \\
\hline Lee [59] & Retrospective, observational & Pseudo-healthy & Lumbar & 44 & 9 & Yes \\
\hline Lundberg [57] & Prospective, case-control & Pseudo-healthy & Ventricular & 1 & 45 & No \\
\hline Magneli [54] & Prospective, case-control & Pseudo-healthy & Parenchymal & 1 & 8 & Yes \\
\hline Mahr [78] & Prospective, observational & Pseudo-healthy & Parenchymal & 35 & 73 & No \\
\hline Malm [5] & Prospective, observational & Healthy & Lumbar & 40 & 70 & Yes \\
\hline Martin [79] & Prospective, case-control & Pseudo-healthy & Intracranial & 1 & 19 & No \\
\hline Pedersen [41] & Retrospective, observational & Pseudo-healthy & Parenchymal & 35 & $4-85$ & Yes \\
\hline Petersen [55] & Prospective, observational & Pseudo-healthy & Parenchymal and ventricular & 11 & 44 & Yes \\
\hline Puhringer [37] & Prospective, observational & Healthy & Lumbar & 5 & $25-38$ & Yes \\
\hline Purvin [52] & Retrospecitve, case-control & Pseudo-healthy & Lumbar & - & - & No \\
\hline Riedel [42] & Prospective, observational & Pseudo-healthy & Parenchymal & 44 & 60 & Yes \\
\hline Schwartz [58] & Prospective, observational & Pseudo-healthy & Lumbar & 55 & 56 & Yes \\
\hline Shapiro [29] & Prospective, observational & Healthy & Lumbar & 23 & $0-55$ & Yes \\
\hline Skau [30] & Prospective, case-control & Healthy & Lumbar & 20 & - & Yes \\
\hline Skipper [31] & Retrospective, case-control & Healthy & Lumbar & 24 & 33 & No \\
\hline Sugita [64] & Prospective, case-control & Pseudo-healthy & Lumbar & 3 & 38 & No \\
\hline Whiteley [11] & Retrospective, observational & Pseudo-healthy & Lumbar & 242 & - & Yes \\
\hline Wibroe [65] & Prospective, observational & Pseudo-healthy & Lumbar & 28 & 37 & Yes \\
\hline
\end{tabular}

\footnotetext{
a Studies were not included in the analysis, if they provided insufficient stastical data to calculate a mean and standard deviation
} 
Table 2 Reference vales for intracranial pressure and lumbar cerebrospinal fluid pressure in different body positions

\begin{tabular}{|c|c|c|c|c|}
\hline & No. of studies & No. of participants & $\begin{array}{l}\text { Mean (reference interval) } \\
{[\mathrm{mmHg}]}\end{array}$ & $\begin{array}{l}\text { Mean (reference } \\
\text { interval) }\left[\mathrm{cmH}_{2} \mathrm{O}\right]\end{array}$ \\
\hline \multicolumn{5}{|l|}{ ICP } \\
\hline Supine & 6 & 62 & $8.6(0.9-16.3)$ & $11.7(1.2-22.2)$ \\
\hline Upright & 6 & 62 & $1.0(-5.9$ to 8.3$)$ & $1.3(-8.7-11.2)$ \\
\hline Daytime & 2 & 45 & $-0.1(-12.0-12.2)$ & $-0.15(-16.3-16.6)$ \\
\hline Nighttime & 3 & 57 & $6.3(-15.8-28.2)$ & $8.6(-21.5-38.3)$ \\
\hline \multicolumn{5}{|l|}{$\mathrm{CSF}_{\mathrm{op}}$} \\
\hline Supine & 7 & 389 & $10.7(5.7-15.5)$ & $14.4(7.5-21.1)$ \\
\hline Lateral recumbent & 21 & 13,359 & $11.9(7.2-16.8)$ & $16.3(9.8-22.8)$ \\
\hline
\end{tabular}

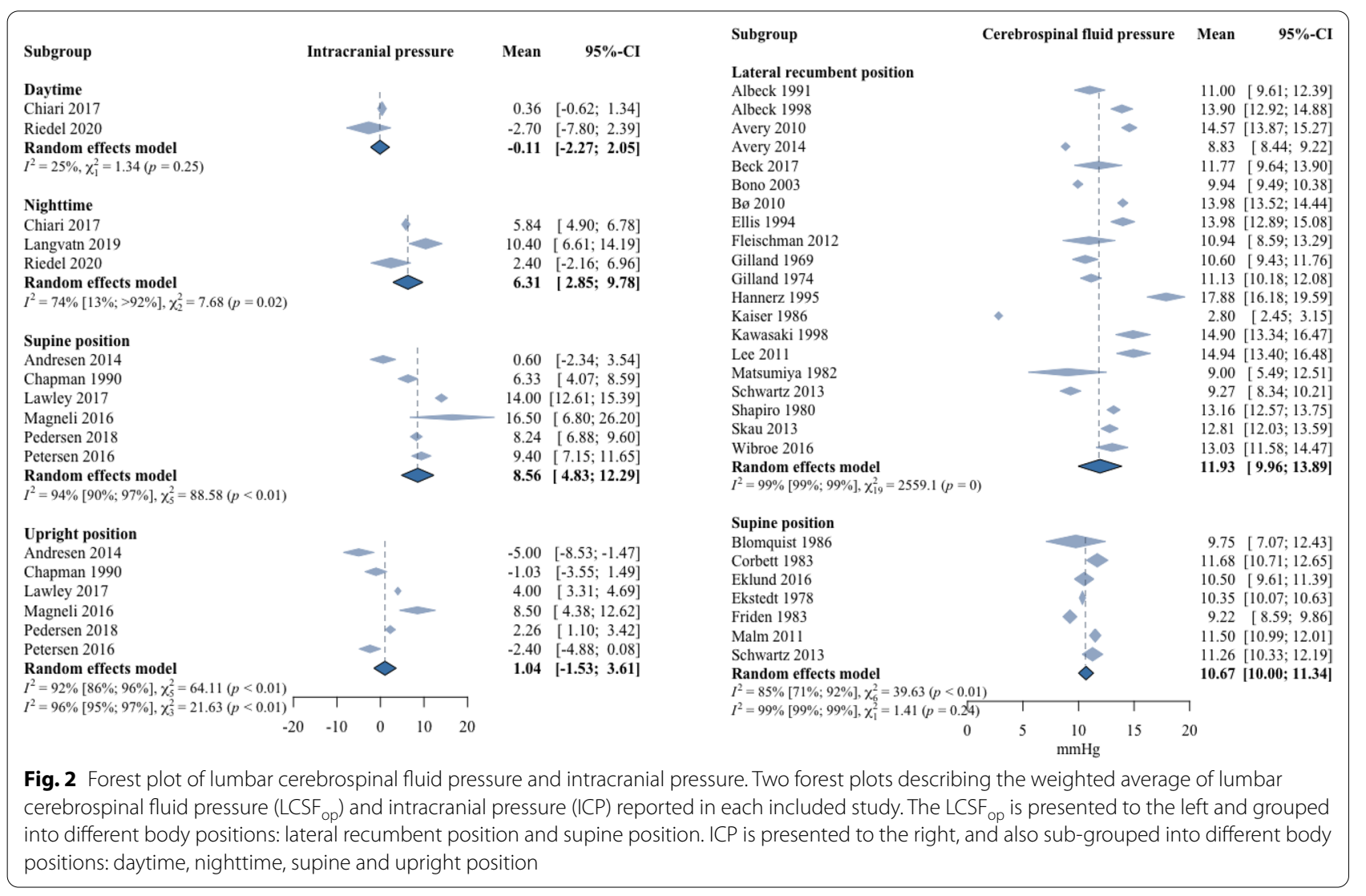

midline, which is a clinical standard in most neurological and neurosurgical departments. However, only nine of the included studies described the zero point of measurements. In terms of the studies reporting on measurement in upright position only two out of six studies reported on zero point. Thus, for the purpose in this systematic review, this was too few, and the reference values were not corrected for zero point.
Although included studies did not provide detailed information regarding age, gender and weight and their influence on ICP, these factors' significance has previously been investigated. Pedersen et al. found ICP to be inversely related to age with an average decrease of $0.69 \mathrm{mmHg}$ per decade [39]. For LCSF ${ }_{\text {op }}$, one study found a steadily decline after 50 years, that females generally had lower LCSF $_{\text {op }}$, and that a high body mass index (BMI) was associated with higher values of $\operatorname{LCSF}_{\mathrm{op}}$ [41] Hannerz et al. and Whiteley et al. 
among many other studies, likewise, reported that ICP or $\operatorname{LCSF}_{\text {op }}$ was higher in obese patients [9, 32, 43-45]. However, the clinical significance of this correlation has been subject to some debate $[9,43]$.

\section{Reference intervals for ICP}

Due to the still invasive nature of obtaining ICP values, it is ethically unacceptable to examine ICP in healthy normal individuals. Several non-invasive ICP measuring methods have been developed, and though they can be useful to estimate interpatient ICP differences, e.g., during follow-up, none are currently accurate enough to measure absolute ICP values $[46,47]$. The studies in this review included pseudo-healthy patients [20,48-50], and we identified no published studies of ICP on completely healthy individuals. A new telemetric monitoring technique has become available in the last decade, allowing for ICP measurements for several months after implantation [51-53]. This has resulted in ICP measurements in groups outside of the traditional patient categories with suspected ICP disorders. Andresen et al. implanted a telemetric ICP monitor in patients who had a small demarcated intracranial tumor surgically removed, thus establishing a pseudo-healthy cohort [48]. Though this cohort is not completely healthy, long-term measurements via the telemetric ICP monitor provide ICP values at a time when intracranial conditions, including ICP, can be considered normal. This is an improvement, since previous research is mainly based on patients undergoing diagnostic evaluation for a suspected ICP disorder [20, $39,54,55]$. Future research to establish normal ICP, until quantitative non-invasive technology is developed, could benefit from this telemetric monitoring technique and, hopefully, expand the material on ICP values obtained in pseudo-healthy humans. However, there are considerable issues concerning spontaneous drift in baseline pressures with the telemetric technique. Drift may occur at any time after implantation. In a previous study, we reported the median drift to be $2.5 \mathrm{mmHg}$ after a median implantation time of 241 days. This study, furthermore, suggested that especially reimplantation of a new sensor into the burr hole of a previous telemetric ICP sensor might be related to measurement errors including drift in baseline pressures [51]. Thus, in future studies on the subject, we recommend (1) a new burr hole for the telemetric ICP sensor, and (2) that the telemetric sensors are tested for zero drift after explantation.

Telemetric ICP monitoring requires implantation of a telemetric ICP sensor. The implantation is comparable to implantation of a cable-based ICP sensor. It does, however, limits the need for repeated invasive procedures (e.g. repeated ICP measurements with a cable-based ICP sensor). In future research of normal ICP, the challenge will thus be to investigate ICP in patients who are as normal as possible in terms of ICP dynamics but still require neurosurgical intervention. A possible cohort could be patients undergoing neurosurgery with removal of a benign intracranial tumor or with clipping of an unruptured aneurysm. Another cohort could be patients with a severe head trauma, who after a full recovery, can be considered without damage to CSF pathways and ICP dynamics $[52,53,56]$.

In this review, we found a reference interval for ICP in the supine position from 0.9 to $16.3 \mathrm{mmHg}$, based on six studies with a total of 62 participants [20, 48, 54, 57-59]. The reference intervals are based on a small sample size, and do, therefore, not represent a clinically-applicable reference interval. Since most humans, and in terms of patients specifically patients with idiopathic intracranial hypertension and normal pressure hydrocephalus, spend the majority of their lives in the upright (vertical) position, a reference interval for this specific position is needed for interpreting diagnostic long-term ICP monitoring. Based on six studies with a total of 62 participants [20, 39, 48, 57-59], this review found a reference intervals for ICP in the upright position from -6.2 to $8.0 \mathrm{mmHg}$. Though too wide to serve as a clinically-applicable reference interval, the data show that negative ICP values can be normal in an upright position $[48,60]$.

For daytime ICP, the established reference intervals were likewise based on a limited number of studies $(\mathrm{n}=2)$ and study participants $(\mathrm{n}=45)[42,61]$. Furthermore, there are large interpersonal differences in the amount of time spend in an upright position during the day. These factors probably result in the wide reference interval from -12.0 to $12.2 \mathrm{mmHg}$. As with the reference interval for ICP in the upright position, this does not serve as a practical tool in clinical decision making. For night-time, we were able to establish a reference interval from -15.8 to $28.2 \mathrm{mmHg}$. Besides a limited number of studies $(\mathrm{n}=3)$ and participants $(\mathrm{n}=57)$ addressing the matter [42, 61, 62], body position during sleep may vary considerably among patients. Furthermore, the degree of sleep apnoea has also been found to highly impact ICP [42]. Combined, this could potentially cause significant variations in measured ICP and, result in the very wide and not clinically useful reference interval. Unfortunately, the included studies did not provide information on body position during sleep or sleep apnoea coherent to ICP values.

There probably is considerable interpersonal differences in normal ICP. Thus, what will be normal in a 10 year-old normal weight girl may differ from an 80 year-old obese man. Therefore, the idea of using a single value of ICP as a way to distinguish normal values from pathological values, is possibly not biologically 
natural. Since ICP is highly dependent on body position and activities, one needs to consider if the values are achieved from bed-bound or active patients. Included data is currently to scarce to make a model accounting for these factors. Instead, we suggest a standardized controlled setting defining "baseline ICP" in different body positions related to patient characteristics (such as age, gender and weight), e.g. 10 min measurement following 20 min supine position.

\section{Reference intervals for $\mathrm{LCSF}_{\text {op }}$}

The majority of included studies on $\operatorname{LCSF}_{\mathrm{op}}(81 \%)$ performed pressure measurement in a lateral recumbent position $[7,9,22,27,28,30-35,41,43,63-73]$, and a reference interval in this position from 7.2 to $16.8 \mathrm{mmHg}$ was established. There was no significant difference between the reference intervals in healthy individuals versus pseudo-healthy individuals. The established reference interval is similar to the reference of 7 to $15 \mathrm{mmHg}$ routinely used in clinical practice [5, 17, 22, 23, 48]. Seven studies obtained LCSF $_{\text {op }}$ values in supine position $[5,7,23,29,63,74,75]$, resulted in a reference interval from 5.7 to $15.5 \mathrm{mmHg}$. Participants in three out of the seven studies were completely healthy $[5,23,29]$. There was no significant difference between reference intervals in healthy individuals versus pseudo-healthy individuals. When comparing supine LCSF $_{\text {op }}$ to supine ICP, we found a difference between the means corresponding to $2.1 \mathrm{mmHg}(\mathrm{P}=0.03)$. These results suggest that supine $\mathrm{LCSF}_{\text {op }}$ is not optimal as a surrogate marker of supine ICP. To our knowledge, there are no studies that compare simultaneously measured supine ICP with supine $\mathrm{LCSF}_{\mathrm{op}}$.

\section{Posture-dependent pressure differences}

Postural-dependent pressure changes have mainly been evaluated in studies investigating ICP $[18,58]$. Andresen et al. compared lumbar recumbent position to supine position in 31 patients with intracranial ICP monitoring. They found that ICP increased approximately $5 \mathrm{mmHg}$ in the lateral recumbent position, a significantly higher difference than found between $\mathrm{LCSF}_{\text {op }}$ in recumbent position versus supine position in this review [18]. This postural-related difference in ICP may be caused by spine flexion and, in particular, flexion of the neck during lateral recumbent position in the study by Andresen et al. [18]. A flexed neck could theoretically compress the jugular veins, thus hindering the venous return to the heart from the head, thereby increasing ICP [40, 76-78]. Many studies have emphasized the importance of a neutral neck during the measurement of $\operatorname{LCSF}_{\text {op }}[22,23,40,76]$. Though statistically significant, the mean $\mathrm{LCSF}_{\text {op }}$ difference of $1.2 \mathrm{mmHg}$ between the lateral recumbent position and supine position hardly has any clinical implications (Fig. 2). This relatively small difference might be explained by $\mathrm{LCSF}_{\mathrm{op}}$ in the lateral recumbent position having been measured with the neck in a neutral position. Unfortunately, the significant change in LCSF $_{\text {op }}$ caused by neck position alone has not previously been subject to much debate. Thus, only six of the included studies examining $\mathrm{LCSF}_{\mathrm{op}}$ documented that $\mathrm{LCSF}_{\mathrm{op}}$ was obtained simultaneously with a neutral neck position $[6,23,27$, $30,33,43]$. To establish an accurate reference interval of $\mathrm{LCSF}_{\mathrm{op}}$, the neck should be held in a neutral position during all $\mathrm{LCSF}_{\mathrm{op}}$ measurements.

\section{Conclusions}

In this systematic review, we aimed to establish reference intervals for ICP and LCSF ${ }_{\mathrm{op}}$.

The data on ICP was not sufficient to establish clinically applicable reference intervals for either supine position, upright position, daytime, or night-time. Negative ICP in upright position do however seem to be normal. For LCSF $_{\text {op }}$ we estimated clinically-applicable reference intervals in both lateral recumbent position (6.3 to $15.9 \mathrm{mmHg}$ ) and supine position (5.3 to $15.1 \mathrm{mmHg}$ ). This systematic review highlights the need for future research within the field of reference intervals for ICP measures.

\section{Supplementary Information}

The online version contains supplementary material available at https://doi. org/10.1186/s12987-021-00253-4.

Additional file 1: Appendix 1. PRISMA checklist.

Additional file 2: Appendix 2. Search strategy.

Additional file 3: Appendix 3. Quality assessment.

\section{Acknowledgements}

Not applicable.

\section{Authors' contributions}

NHN MHO, SSP, CSR, MJ designed the study. NHN, MHO, SSP, CSR screened and extracted data from studies. $\mathrm{NHN}$ and $\mathrm{MHO}$ drafted the manuscript. All authors read and approved the final manuscript.

\section{Funding}

The project was funded by the Rigshospitalet Research Foundation, the DMSK Foundation and the Novo Nordisk Foundation (NNF17OC0024718). The funding bodies had no role in the design of the study, the study collection, analysis, interpretation of data or in writing the manuscript.

\section{Availability of data and materials}

The data set used and analysed during the current study is available from the corresponding author on reasonable request.

\section{Declarations}

Ethics approval and consent to participate Not applicable. 


\section{Consent for publication \\ Not applicable.}

\section{Competing interests}

The authors declare they have no competing interests.

\begin{abstract}
Author details
${ }^{1}$ Department of Neurosurgery, Rigshospitalet, Copenhagen, Denmark.

${ }^{2}$ Department of Neuroanaesthesiology, Rigshospitalet, Copenhagen, Denmark. ${ }^{3}$ Department of Neurophysiology, Rigshospitalet, Glostrup, Denmark. ${ }^{4}$ Brain Physics Laboratory, Department of Clinical Neurosciences, Addenbrooke's Hospital, University of Cambridge, Cambridge, UK. ${ }^{5}$ Department of Neurosurgery, Aarhus University Hospital, Aarhus, Denmark.
\end{abstract}

Received: 4 January 2021 Accepted: 30 March 2021

Published online: 13 April 2021

\section{References}

1. Sæhle T, Eide PK. Intracranial pressure monitoring in pediatric and adult patients with hydrocephalus and tentative shunt failure: a single-center experience over 10 years in 146 patients. J Neurosurg. 2015;122:1076-86. https://doi.org/10.3171/2014.12.JNS141029.

2. Schuhmann MU, Sood S, McAllister JP, Jaeger M, Ham SD, Czosnyka Z, et al. Value of overnight monitoring of intracranial pressure in hydrocephalic children. Pediatr Neurosurg. 2008;44:269-79.

3. Czosnyka Z, Czosnyka M. Long-term monitoring of intracranial pressure in normal pressure hydrocephalus and other CSF disorders. Acta Neurochir. 2017;159:1979-80.

4. Lilja-Cyron A, Kelsen J, Andresen M, Fugleholm K, Juhler M. Feasibility of telemetric intracranial pressure monitoring in the neuro intensive care unit. J Neurotrauma. 2018;35:1578-86.

5. Malm J, Jacobsson J, Birgander R, Eklund A. Reference values for CSF outflow resistance and intracranial pressure in healthy elderly. Neurology. 2011;76:903-9.

6. Gilland O, Tourtellotte WW, O'Tauma L, Henderson WC. Normal cerebrospinal fluid pressure. J Neurosurg. 1974;40:587-93.

7. Ekstedt J. CSF hydrodynamic studies in man II Normal hydrodynamic variables related to CSF pressure and flow. J Neurol Neurosurg Psychiatry. 1978;41:345-53.

8. Loman J, Myerson A, Goldman D. Effects of alterations in posture on the cerebrospinal fluid pressure. Arch Neurol Psychiatry. 1935;33:1279-95.

9. Whiteley W, Al-Shahi R, Warlow CP, Zeidler M, Lueck CJ. CSF opening pressure: reference interval and the effect of body mass index. Neurology. 2006:67:1690-1.

10. Ekstedt J. CSF hydrodynamic studies in man. J Neurol Neurosurg Psychiatry. 1977:40:105-19.

11. Hodgson JS. Combined ventricular and lumbar puncture in the diagnosis of brain tumor. J Am Med Assoc. 1928;90:1524-6.

12. Smyth GE, Henderson WR. Observations on the cerebrospinal fluid pressure on simultaneous ventricular and lumbar punctures. J Neurol Neurosurg Psychiatry. 1938;1:226-38. https://doi.org/10.1136/jnnp.1.3. 226.

13. Speck V, Staykov D, Huttner HB, Sauer R, Schwab S, Bardutzky J. Lumbar catheter for monitoring of intracranial pressure in patients with post-hemorrhagic communicating hydrocephalus. Neurocrit Care. 2011;14:208-15.

14. Eide PK, Brean A. Lumbar cerebrospinal fluid pressure waves versus intracranial pressure waves in idiopathic normal pressure hydrocephalus. Br J Neurosurg. 2006;20:407-14.

15. Lenfeldt N, Koskinen LOD, Bergenheim AT, Malm J, Eklund A. CSF pressure assessed by lumbar puncture agrees with intracranial pressure. Neurology. 2007;68:155-8.

16. Warden KF, Alizai AM, Trobe JD, Hoff JT. Short-term continuous intraparenchymal intracranial pressure monitoring in presumed idiopathic intracranial hypertension. J Neuro-Ophthalmol. 2011;31:202-5.

17. Eide PK, Due-Tønnessen B, Helseth E, Lundar T. Assessment of intracranial pressure volume relationships in childhood: the lumbar infusion test versus intracranial pressure monitoring. Child's Nerv Syst. 2001;17:382-90.
18. Andresen M, Hadi A, Petersen LG, Juhler M. Effect of postural changes on ICP in healthy and ill subjects. Acta Neurochir. 2014;157:109-13.

19. Poca MA, Sahuquillo J, Topczewski T, Lastra R, Font ML, Corral E. Postureinduced changes in intracranial pressure: a comparative study in patients with and without a cerebrospinal fluid block at the craniovertebral junction. Neurosurgery. 2006;58:899-906.

20. Chapman PH, Cosman ER, Arnold MA, Portnoy HD, Sklar F. The relationship between ventricular fluid pressure and body position in normal subjects and subjects with shunts: a telemetric study. Neurosurgery. 1990;26:181-9.

21. Barami K, Sood S, Ham SD, Canady Al. Postural changes in intracranial pressure in chronically shunted patients. Pediatr Neurosurg. 2000;33:64-9.

22. Ellis R. Lumbar cerebrospinal fluid opening pressure measured in a flexed lateral decubitus position in children. Pediatrics. 1994;24:622-3.

23. Corbett JJ, Mehta MP. Cerebrospinal fluid pressure in normal obese subjects and patients with pseudotumor cerebri. Neurology. 1983;33:1386-8.

24. Wilson TJ. Textbook of child neurology. J Neurol Neurosurg Psychiatry. 1980;43:1050-1.

25. The Joanna Briggs Institute. Joanna Briggs Institute Reviewer's Manual. Joanna Briggs Inst. 2014.

26. Higgins J, Green S. Cochrane handbook for systematic reviews of interventions version 5.1.0. Cochrane Database Syst Rev. 2011.

27. Skau M, Yri H, Sander B, Gerds TA, Milea D, Jensen R. Diagnostic value of optical coherence tomography for intracranial pressure in idiopathic intracranial hypertension. Graefe's Arch Clin Exp Ophthalmol. 2012;251:567-74.

28. Skipper NT, Igra MS, Littlewood R, Armitage P, Laud PJ, Mollan SP, et al. Do optic canal dimensions measured on CT influence the degree of papilloedema and visual dysfunction in idiopathic intracranial hypertension? Neuro-Ophthalmol. 2019;43:3-9.

29. Eklund A, Jóhannesson G, Johansson E, Holmlund P, Qvarlander S, Ambarki $K$, et al. The pressure difference between eye and brain changes with posture. Ann Neurol. 2016;80:269-76.

30. Albeck MJ, Borgesen SE, Gjerris F, Schmidt JF, Sorensen PS. Intracranial pressure and cerebrospinal fluid outflow conductance in healthy subjects. J Neurosurg. 1991;74:597-600.

31. Albeck MJ, Skak C, Nielsen PR, Olsen KS, Børgesen SE, Gjerris F. Age dependency of resistance to cerebrospinal fluid outflow. J Neurosurg. 1998:89:275-8

32. Hannerz J, Greitz D, Ericson K. Is there a relationship between obesity and intracranial hypertension? Int J Obes Relat Metab Disord. $1995 ; 19: 240-4$

33. Lakke JP, Schut D. Electromanometric examination of CSF pressure response to jugular compression. I. Determination of normal values. Psychiatr Neurol Neurochir. 1968;71:57-64.

34. Puhringer F, Hormann C, Langmayr J, Schalow S, Benzer A. The effect of alfentanil on cerebrospinal fluid pressure in human volunteers. Eur J Anaesthesiol. 1997;14:211-4.

35. Shapiro K, Marmarou A, Shulman K. Characterization of clinical CSF dynamics and neural axis compliance using the pressure-volume index: I The normal pressure-volume index. Ann Neurol. 1980;7:508-14.

36. $\mathrm{B} \varnothing \mathrm{SH}$, Lundqvist $\mathrm{C}$. Cerebrospinal fluid opening pressure in clinical practice - a prospective study. J Neurol. 2020;267:3696-701. https://doi. org/10.1007/s00415-020-10075-3.

37. Pedersen $\mathrm{SH}$, Norager $\mathrm{NH}$, Lilja-Cyron A, Juhler M. Telemetric intracranial pressure monitoring in children. Child's Nerv Syst. 2020;36:49-58.

38. Eide PK, Bakken A. The baseline pressure of intracranial pressure (ICP) sensors can be altered by electrostatic discharges. Biomed Eng Online. 2011;10:76.

39. Pedersen SH, Lilja-Cyron A, Andresen M, Juhler M. The relationship between intracranial pressure and age_chasing age-related reference values. World Neurosurg. 2018;110:119-23. https://doi.org/10.1016/j. wneu.2017.10.086.

40. Williams A, Coyne SM. Effects of neck position on intracranial pressure. Am J Crit Care. 1993:2:68-71.

41. Fleischman D, Berdahl JP, Zaydlarova J, Stinnett S, Fautsch MP, Allingham RR. Cerebrospinal fluid pressure decreases with older age. PLoS ONE. 2012;7:1-9.

42. Riedel CS, Martinez-tejada I, Norager NH, Juhler M. B-waves are present in patients without intracranial pressure disturbances. J Sleep Res. 2020;517. 
file:///C:/Oel/Artikler/PhD-Mxa-2. package (clinmon)/Riedel et al. B-waves manuscript_sleep_research_vers3.1.pdf

43. Bono F, Lupo MR, Lavano A, Mangone L, Fera F, Pardatscher K, et al. Cerebral MR venography of transverse sinuses in subjects with normal CSF pressure. Neurology. 2003;61:1267-70.

44. Berdahl JP, Fleischman D, Zaydlarova J, Stinnett S, Rand Allingham R, Fautsch MP. Body mass index has a linear relationship with cerebrospinal fluid pressure. Investig Ophthalmol Vis Sci. 2012;53(3):1422-7.

45. Wakerley BR, Warner R, Cole M, Stone K, Foy C, Sittampalam M. Cerebrospinal fluid opening pressure: the effect of body mass index and body composition. Clin Neurol Neurosurg. 2020;188:105597.

46. Raboel PH, Bartek J, Andresen M, Bellander BM, Romner B. Intracranial pressure monitoring: Invasive versus non-invasive methods-A review. Crit Care Res Pract. 2012;2012:3-7.

47. Kawoos U, McCarron RM, Auker CR, Chavko M. Advances in intracranial pressure monitoring and its significance in managing traumatic brain injury. Int J Mol Sci. 2015;16:28979-97.

48. Andresen M, Juhler M. Intracranial pressure following complete removal of a small demarcated brain tumor: a model for normal intracranial pressure in humans. J Neurosurg. 2014;121:797-801. https://doi.org/10.3171/ 2014.2.JNS132209.

49. Petersen LG, Lawley JS, Lilja-Cyron A, Petersen JCGG, Howden EJ, Sarma S, et al. Lower body negative pressure to safely reduce intracranial pressure. J Physiol England. 2019;597:237-48.

50. Magnéli S, Howells T, Saiepour D, Nowinski D, Enblad P, Nilsson P. Telemetric intracranial pressure monitoring: a noninvasive method to follow up children with complex craniosynostoses. A case report. Child's Nerv Syst Child's Nervous System. 2016;32:1311-5. https://doi.org/10.1007/ s00381-016-3023-4.

51. Norager NH, Lilja-cyron A, Bjarkam CR, Duus S, Juhler M. Telemetry in intracranial pressure monitoring : sensor survival and drift. Acta Neurochir. 2018;160:2137-44.

52. Norager NH, Lilja-Cyron A, Hansen TS, Juhler M. Deciding on appropriate telemetric intracranial pressure monitoring system. World Neurosurg. 2019;126:564-9. https://doi.org/10.1016/j.wneu.2019.03.077.

53. Antes S, Tschan CA, Heckelmann M, Breuskin D, Oertel J. Telemetric intracranial pressure monitoring with the raumedic neurovent P-tel. World Neurosurg. 2016;91:133-48. https://doi.org/10.1016/j.wneu.2016.03.096.

54. Purvin VA, Kawasaki A, Yee RD. Papilledema and obstructive sleep apnea syndrome. Arch Ophthalmol. 2000;118:1626-30.

55. Lundberg N. Continuous recording and control of ventricular fluid pressure in neurosurgical practice. Acta Psychiatr Scand Suppl. 1960;36:1-193.

56. Antes S, Stadie A, Müller S, Linsler S, Breuskin D, Oertel J. Intracranial Pressure-Guided shunt valve adjustments with the miethke sensor reservoir. World Neurosurg. 2018;109:e642-50.

57. Magnéli S, Howells T, Saiepour D, Nowinski D, Enblad P, Nilsson P. Telemetric intracranial pressure monitoring: a noninvasive method to follow up children with complex craniosynostoses. A case report. Child's Nerv Syst. 2016;32:1311-5.

58. Petersen LG, Petersen JCG, Andresen M, Secher NH, Juhler M. Postural influence on intracranial and cerebral perfusion pressure in ambulatory neurosurgical patients. Am J Physiol Regul Integr Comp Physiol. 2016:310:R100-4. https://doi.org/10.1152/ajpregu.00302.2015.

59. Lawley JS, Petersen LG, Howden EJ, Sarma S, Cornwell WK, Zhang R, et al. Effect of gravity and microgravity on intracranial pressure. J Physiol. 2017;595:2115-27.

60. Lundberg N. The mean ventricular fluid pressure. Acta Psychiatr Scand Suppl. 1960;36:1-193.
61. Chari A, Dasgupta D, Smedley A, Craven C, Dyson E, Matloob S, et al. Intraparenchymal intracranial pressure monitoring for hydrocephalus and cerebrospinal fluid disorders. Acta Neurochir. 2017;159:1967-78. https:// doi.org/10.1007/s00701-017-3281-2.

62. Langvatn EA, Frič R, Due-Tønnessen BJ, Eide PK. Intracranial volume versus static and pulsatile intracranial pressure values in children with craniosynostosis. J Neurosurg Pediatr. 2019;24:66-74.

63. Schwartz KM, Luetmer PH, Hunt CH, Kotsenas AL, Diehn FE, Eckel LJ, et al. Position-related variability of CSF opening pressure measurements. Am J Neuroradiol. 2013;34:904-7.

64. Lee MW, Vedanarayanan VV. Cerebrospinal fluid opening pressure in children: experience in a controlled setting. Pediatr Neurol. 2011;45:238-40.

65. Kaiser AM, Whitelaw AGL. Normal cerebrospinal fluid pressure in the newborn. Neuropediatrics. 1986;17:100-2.

66. Kawasaki A, Purvin V. Unilateral optic disc edema following trabeculectomy. J Neuro-Ophthalmol. 1998;18:121-3.

67. González-Camarena PI, San-Juan D, González-Olhovich I, RodríguezArévalo D, Lozano-Elizondo D, Trenado C, et al. Dynamic changes of the intraocular pressure and the pressure of cerebrospinal fluid in nonglaucomatous neurological patients. Acta Ophthalmol. 2017;95:e138-43.

68. Avery RA, Shah SS, Licht DJ, Seiden JA, Huh JW, Boswinkel J, et al. Reference range for cerebrospinal fluid opening pressure in children. $N$ Engl J Med. 2010;363:891-3.

69. Sugita Y, lijima S, Teshima Y, Shimizu T, Nishimura N, Tsutsumi T, et al. Marked episodic elevation of cerebrospinal fluid pressure during nocturnal sleep in patients with sleep apnea hypersomnia syndrome. Electroencephalogr Clin Neurophysiol. 1985;60:214-9.

70. Wibroe EA, Yri HM, Jensen RH, Wibroe MA, Hamann S. Osmolality of cerebrospinal fluid from patients with idiopathic intracranial hypertension (IIH). PLOS ONE. 2016;11:e0146793.

71. Beck J, Fung C, Ulrich CT, Fiechter M, Fichtner J, Mattle HP, et al. Cerebrospinal fluid outflow resistance as a diagnostic marker of spontaneous cerebrospinal fluid leakage. J Neurosurg Spine. 2017;27:227-34.

72. Gulbrandsen P, Dietrichs E. Cerebrospinal fluid opening pressure measurements in acute headache patients and in patients with either chronic or no pain. Acta Neurol Scand. 2010;122:6-11.

73. Avery RA. Interpretation of lumbar puncture opening pressure measurements in children. J Neuro-Ophthalmol. 2014;34:284-7.

74. Friden HG, Ekstedt J. Volume/pressure relationship of the cerebrospinal space in humans. Neurosurgery. 1983;13:351-66.

75. Blomquist HK, Sundin S, Ekstedt J. Cerebrospinal fluid hydrodynamic studies in children. J Neurol Neurosurg Psychiatry. 1986;49:536-48.

76. Hulme A, Cooper R. The effects of head position and jugular vein compression (JVC) on intracranial pressure (ICP). A Clin Study Intracranial Press. 1976; III:259-63.

77. Lipe HP, Mitchell PH. Positioning the patient with intracranial hypertension: how turning and head rotation affect the internal jugular vein. Hear Lung. 1980;9:1031-7.

78. Holmlund P, Eklund A, Koskinen LOD, Johansson E, Sundström N, Malm J, et al. Venous collapse regulates intracranial pressure in upright body positions. Am J Physiol Regul Integr Comp Physiol. 2018;314:R377-85.

79. Martin G. Lundberg's B waves as a feature of normal intracranial pressure. Surg Neurol. 1978;9(6):347-8.

\section{Publisher's Note}

Springer Nature remains neutral with regard to jurisdictional claims in published maps and institutional affiliations. 